\title{
Migração Kirchhoff modificada em profundidade utilizando contribuições paraxiais em uma geologia complexa
}

Manuel J. S. Costa, Universidade Federal do Pará - UFPA

João Carlos R. Cruz, Universidade Federal do Pará - UFPA

Paulo Eduardo Miranda Cunha, Petróleo Brasileiro S.A - Petrobas

Luis Eduardo S. Santos, Universidade Federal do Pará - UFPA

Copyright 2018, SBGf - Sociedade Brasileira de Geofísica

Este texto foi preparado para a apresentação no VIII Simpósio Brasileiro de Geofísica, Salinópolis, 18 a 20 de setembro de 2018. Seu conteúdo foi revisado pelo Comitê Técnico do VIII SimBGf, mas não necessariamente representa a opinião da SBGf ou de seus associados. É proibida a reprodução total ou parcial deste material para propósitos comerciais sem prévia autorização da SBGf.

\begin{abstract}
This work considers 2D true amplitude modified Kirchhoff type prestack depth migration in the common offset domain. The appoach considers the superposiion of paraxial amplitudes in the vicinity of every trace location in a common offset section, mapped throgh the determination of the Fresnel zone elements of the seismic experimente. These amplitudes are the stacked and smeared by the use paraxial operador, which is a highfrequency representation of the seismic wave field, prior to being migrated in th common offset domain to is true position in the depth domain.
\end{abstract}

\section{Introdução}

Métodos de imageamento sísmico aplicados a dados sísmicos de reflexão objetivam colapsar as difrações posicionando corretamente os eventos de reflexão em suas verdadeiras posições espaciais. Para exploração de hidrocarbonetos considerando a existência de um modelo de velocidade acurado obtido via processamento de dados o objetivo principal é produzir as melhores imagens possíveis dos refletores em subsuperfície para auxiliar interpretes no mapeamento das interfaces estruturais que separam formações geológicas contendo óleo e que podem representar armadilhas para acumulações de petróleo e gás. Entre os métodos de imageamento sísmico utilizados pela indústria petrolífera, os métodos do tipo Kirchhoff, bem como os baseados na equação da onda são os mais representativos cada um com suas próprias vantagens e desvantagens. Esses diferentes métodos de imageamento podem ser aplicados a certas classes de classificação ordenada: pós-empilhamento e pré-empilhamento, 2D ou 3D. O resultado final (imagens migradas em subsuperfície) pode ser exibido em profundidade ou no tempo. Neste sentido, considerando a necessidade contínua de melhorar a resolução das imagens sísmicas, o método a ser abordado neste trabalho refere-se a uma migração modificada pré-empilhamento em profundidade $2 \mathrm{D}$ do tipo Kirchhoff. O propósito do operador integral de migração proposto foi modificado para inserir em seu núcleo uma aproximação do campo de ondas paraxial representada por uma binagem, tal aproximação funciona da mesma forma que a função de Green do problema de imageamento no domínio em particular afastamentocomum (Ferreira e Cruz, 2009), levando em consideração a fundamentação teórica de amplitudes verdadeiras (Schleicher et al., 1993; Tygel et al., 1996), objetivando atenuar os efeitos do espalhamento geométrico nas amplitudes de reflexão. Nesta migração, aplica-se um processo em cascata, isto é, inicialmente faz-se uma superposição de contribuições paraxiais janelados pela zona de Fresnel projetada utilizando um operador de empilhamento parabólico definido em função de um traço especular a ser migrado com a curva de difração $\tau_{D}(\xi)$ (curva de Huygens). Um exemplo numérico será mostrado, utilizando um dado sísmico oriundo de uma geologia complexa (Marmousi) mostrando a eficácia desta nova abordagem.

\section{Metodologia}

A forma geral do operador de migração do tipo Kirchhoff em 2D é dada por (Schleicher et al., 1993):

$$
I(R)=\int_{A} d \xi w(\xi, R) D\left[U\left(\xi, t=\tau_{D}(\xi, R)\right)\right](1)
$$

onde, $I(R)$ denota a amplitude atribuída ao ponto de difração $R$ no domínio migrado, $U(\xi, t)$ representa os traços sísmicos da seção de entrada descritos pelo parâmetro de configuração $\xi$, cuja variação ocorre dentro de uma abertura de migração $A$, ponderada pela função peso $w(\xi, R)$, com $D$ representando uma meia derivada aplicada aos dados para recuperar o pulso fonte após o processo de migração. Neste contexto, salientase que a função peso supracitada procura remover da amplitude migrada, o efeito do espalhamento geométrico, caracterizando assim, uma migração do tipo Kirchhoff em verdadeira amplitude.

\section{Migração Kirchhoff modificada}

Seguindo a fundamentação teórica da migração proposta em Ferreira e Cruz (2005), Sun et al. (2000), Costa (2012) e Garabito et al. (2012) o núcleo do operador de migração (1) é modificado através de uma superposição de contribuições na vizinhança de um raio de referência especular, através de um empilhamento ao longo da curva parabólica (ver, 6) confinado em um tamanho 
determinado pela zona de Fresnel projetada $\left(H_{P}\right)$, onde a mesma calcula-se por (Costa, 2012):

$$
H_{P}=\frac{\Lambda^{2}}{H_{F}}
$$

sendo,

$$
\Lambda=\left(-\frac{Q_{2, R G}}{\cos \beta_{G} \cos \beta_{S}}\right)^{-1} \Gamma_{G}+\left(-\frac{Q_{2, S R}}{\cos \beta_{G} \cos \beta_{S}}\right)^{-1} \Gamma_{S}(3)
$$

e

$$
H_{F}=\frac{Q_{1, R G}}{Q_{2, R G}} \cos ^{2} \beta_{S}+\frac{P_{2, S R}}{Q_{2, S R}} \cos ^{2} \beta_{G} .
$$

Nesta abordagem, $\Gamma_{S}, \Gamma_{G}$ são escalares a especificar dependendo da configuração sísmica, $\beta_{S}$ e $\beta_{G}$ correspondem aos ângulos que o raio central forma com a normal da linha de aquisição, $P_{2}, Q_{1}$ e $Q_{2}$ elementos escalares que caracterizam o raio central, os quais são oriundos do sistema de traçamento dinâmico do raio (TDR), $S R$ e $R G$ caracterizam trajetórias dos raios que ligam a fonte $S$ a um ponto $R$ e este ao receptor $G$. Assim, o empilhamento interno supracitado para um determinado tempo de difração $\tau_{D}$ do traço de referência $\xi_{D}$ pertencente a uma janelamento local dos dados de entrada $U(\xi, t)$, define-se como segue:

$$
\hat{U}\left(\xi_{D}, \tau_{D}\right)=\int_{A_{P}} d \xi_{p} \sqrt{H_{P}\left(\xi_{P}\right)} L\left(\xi_{P}\right) U\left(\xi_{P}, T_{P}\left(\xi_{P}, \xi_{D}\right)\right)
$$

onde, $\sqrt{H\left(\xi_{P}\right)}$ determina a função peso do empilhamento interno dentro da zona de Fresnel projetada, a qual vincula a abertura de migração $A_{P}, \xi_{P}$ representa a posição dos pontos na vizinhança do traço sísmico de referência $\xi_{D}, L\left(\xi_{P}\right)$ o decaimento da amplitude do dado sísmico no interior do empilhamento centrado na coordenada de referência e $T_{P}\left(\xi_{P}, \xi_{D}\right)$ representando a curva parabólica relativa que coleta as contribuições paraxiais. Destaca-se que nesta nova estratégia de migração $T_{P}\left(\xi_{P}, \xi_{D}\right)$ é definido por:

$T_{P}\left(\xi_{P}, \xi_{D}\right)=\tau_{D}\left(\xi_{D}\right)+p\left(\xi_{P}-\xi_{D}\right)+\frac{1}{2} H_{P}\left(\xi_{P}\right)\left(\xi_{P}-\xi_{D}\right)^{2}(6)$

com $p$ sendo a vagarosidade horizontal do raio de referência e $\tau_{D}\left(\xi_{D}\right)$ determinando o tempo de trânsito da curva de difração (curva de Huygens).

\section{Interpretação geométrica da superposição de contribuições paraxiais}

Para um melhor entendimento da contribuição paraxial quando se utiliza (5), observe a Figura (1), onde a curva de difração $\tau_{D}\left(\xi_{D}\right)$ é a curva de empilhamento de Huygens do operador de migração (1). A vagarosidade horizontal $p($ ver, 6) determina a direção dos eventos chamada de direção "slant" ou direção oblíqua, $H_{P}\left(\xi_{P}\right)$ (ver, 6) é responsável pela curvatura local dos eventos paraxiais ao redor do traço de referência $\xi_{D}$. Sendo assim, (5) sobre a abertura de migração $A_{P}$ empilha os referidos eventos em $\xi_{D}$, caracterizando assim um empilhamento oblíquo local.

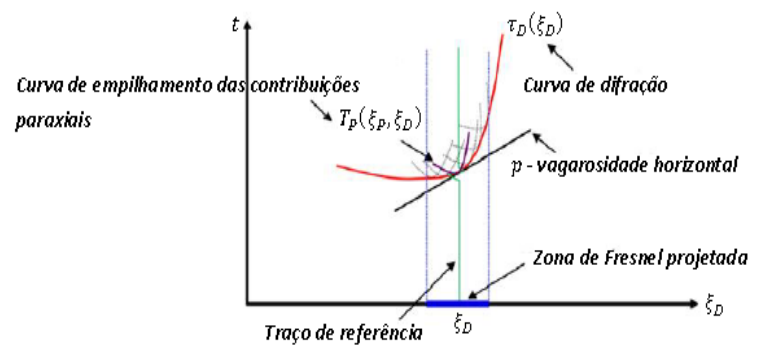

Figura 1- Representação esquemática 2D do processo de empilhamento das amplitudes utilizando aproximações paraxiais.

\section{Operador de migração Kirchhoff modificado no domínio afastamento comum (KM-AC)}

Será apresentado um operador de migração Kirchhoff modificado em seu núcleo, através de uma aproximação do campo de ondas por uma superposição de contribuições paraxiais (ver, 5). Neste contexto, o operador (1) modificado será definido na configuração de medida afastamento comum (AC), como segue (Costa, 2012):

$$
\begin{aligned}
& I(R)=-\frac{1}{2 \pi} \int_{A} d x_{m} w\left(x_{m}, R\right) \int_{A_{P}} \sqrt{H_{P}\left(x_{m}^{P}\right)} D_{L}\left(x_{m}^{P}\right) \\
& D^{*}\left[U\left(x_{m}, t=\tau_{D}\left(x_{m}, x_{m}^{P}, R\right)\right)\right]
\end{aligned}
$$

onde, $x_{m}$ representa a coordenada ponto médio comum denotando a localização dos traços a serem migrados pela curva de Huygens, sendo a variável de integração com abertura $A, x_{m}^{P}$ denota as posições dos traços pertencentes a abertura $A_{P}, R$ indica uma identidade com um ponto de reflexão em profundidade, $w\left(x_{m}, R\right)$ e $\sqrt{H_{P}\left(x_{m}^{P}\right)}$ funções pesos no processo, 
$\tau_{D}\left(x_{m}, x_{m}^{P}, R\right)$ correspondendo a curva relativa de difração que coleta as contribuições paraxiais, $D^{*}$ um processo duplo de meia derivada e $D_{L}\left(x_{m}^{P}\right)$ caracterizando uma função tipo taper.

\section{Principais passos do algoritmo KM-AC}

Nesta seção serão listadas as etapas que implementam a migração KM-AC:

I- Entrada:

- Volume do dado sísmico com cobertura múltipla organizado em famílias afastamento comum (AC);

II- Para cada ponto imagem da seção de saída, calcular:

- O ponto médio, meio afastamento e a curva de difração $\mathrm{CO}$, bem como a função peso associada à curva de difração AC;

IV- Tempos da curva de difração:

- Para cada tempo das curvas de difração AC, calcular a curva paraxial no domínio $A C$, os parâmetros que definem a abertura e 0 peso das superposições das contribuições paraxiais no domínio AC, em seguida fazer o empilhamento dos traços paraxiais;

\section{V- Somando amplitudes:}

- Somam as amplitudes advindas da soma paraxial ao longo da curva de difração $\mathrm{AC}$, repetindo o procedimento para todos os pontos de amostragem das seções de saída;

VI- Saída:

- Seções migradas para afastamento comum; seção migrada empilhada final.

\section{Resultados numéricos}

O conjunto de dados Marmousi utilizado para os experimentos numéricos possui 261 tiros, cada seção de tiro comum com 96 receptores, apresentando um afastamento mínimo entre fonte-receptor de $100 \mathrm{~m}$. Fontes e receptores estão regularmente espaçados de $25 \mathrm{~m}$. A amostragem do dado é de 4 milissegundos (ms) com tempo de aquisição de 3 segundos (s). O modelo de velocidade original do Marmousi é visualizado na Figura (2). Destaca-se que o dado em questão apresenta ausência de múltiplas em virtude da modelagem utilizar a aproximação de Born. Para efeito de visualização é mostrado na Figura (3) a seção de afastamento comum $200 \mathrm{~m}$. Neste sentido, inicialmente será apresentado à resposta do operador (6) levando em consideração uma janela contendo cinco traços de referência da seção afastamento comum $200 \mathrm{~m}$ (ver, Figura 4), também apresentar-se-á uma imagem migrada final (ver, Figura 5) utilizando todos os afastamentos comuns referente ao dado sísmico Marmousi (VERSTEEG, 1994). Será apresentada ainda famílias de imagem comum (do inglês,
CIGs) correspondente a posições no dado sísmico, isto é $4000 \mathrm{~m}, 4025 \mathrm{~m}$ e $4050 \mathrm{~m}$, respectivamente (ver, Figura 6). Por fim, um exemplo mostrando como o processo KM-AC se comporta na presença de ruído aleatório com razão/sinal ruído igual a 3 aplicado aos dados sísmicos. Para ilustrar tal processo, visualiza-se a Figura (7) como um dado de entrada (seção afastamento comum $200 \mathrm{~m}$ ) e a Figura (8) como a seção migrada final nesta situação, isto é utilizando todos os afastamentos contaminados com ruído aleatório aditivo.

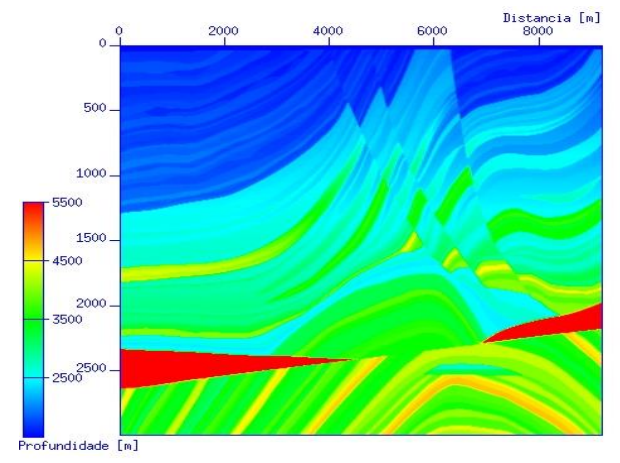

Figura 2- Modelo de velocidade compressional Marmousi

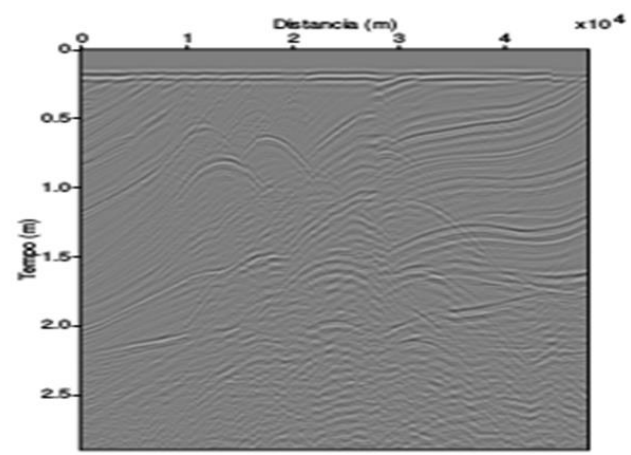

Figura 3- Seção sísmica de afastamento comum $200 \mathrm{~m}$

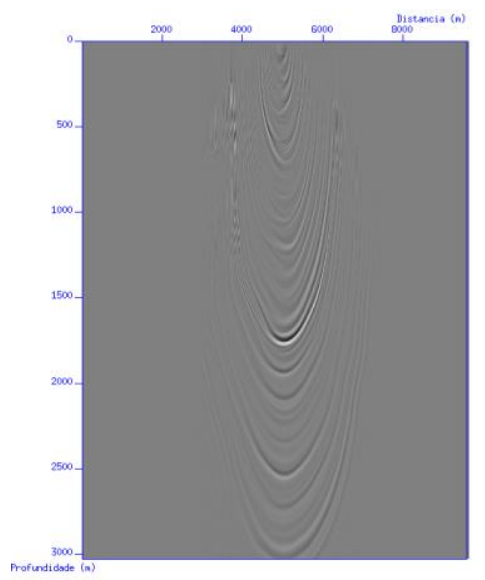

Figura 4- Resposta do operador KM-AC aplicado sobre cinco traços de referência retirados do afastamento mínimo $100 \mathrm{~m}$. 


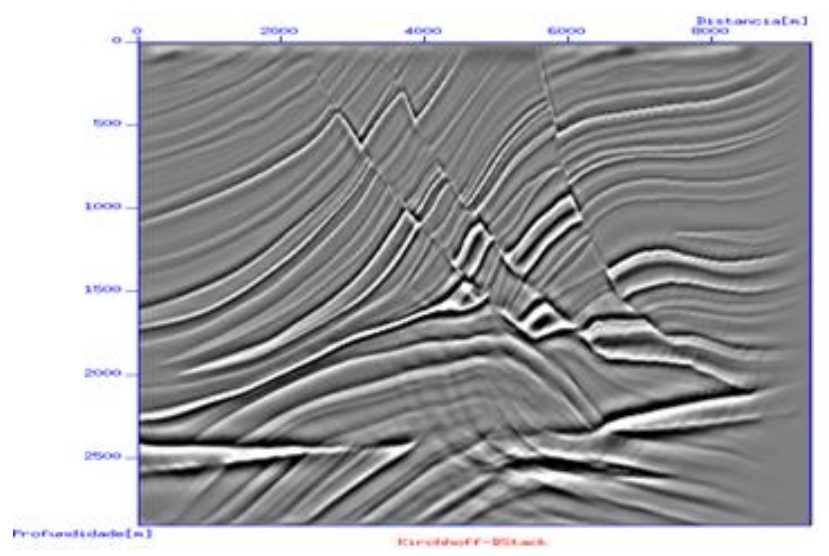

Figura 5- Seção migrada final resultante do empilhamento de todos os afastamentos comuns migrados do dado sísmico Marmnııi

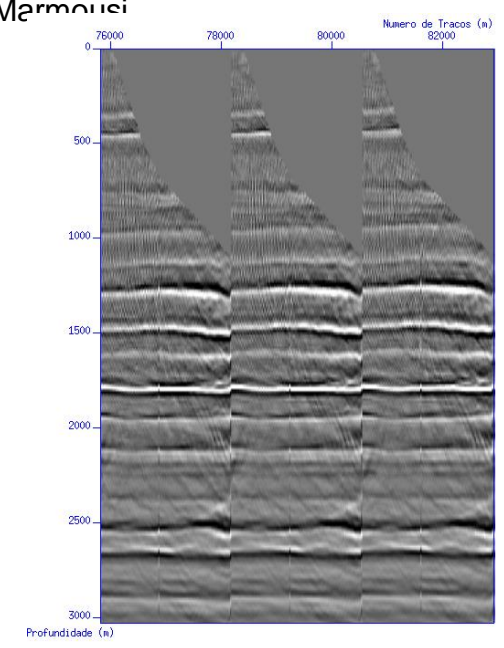

Figura 6- Famílias de imagem comum (CIGs) resultante da migração $\mathrm{KM}-\mathrm{AC}$. $\mathrm{O}$ três CIGs correspondem as seguintes posições na linha sísmica, respectivamente: $4000 \mathrm{~m}, 4025 \mathrm{~m}$ e $4050 \mathrm{~m}$.

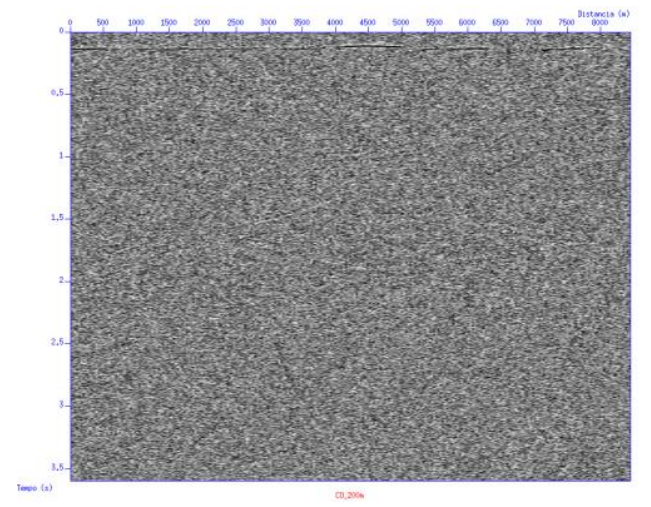

Figura 7- Seção sísmica com afastamento constante $200 \mathrm{~m}$ com ruído aleatório aditivo, onde a razão sinal/ruído é igual a 3.

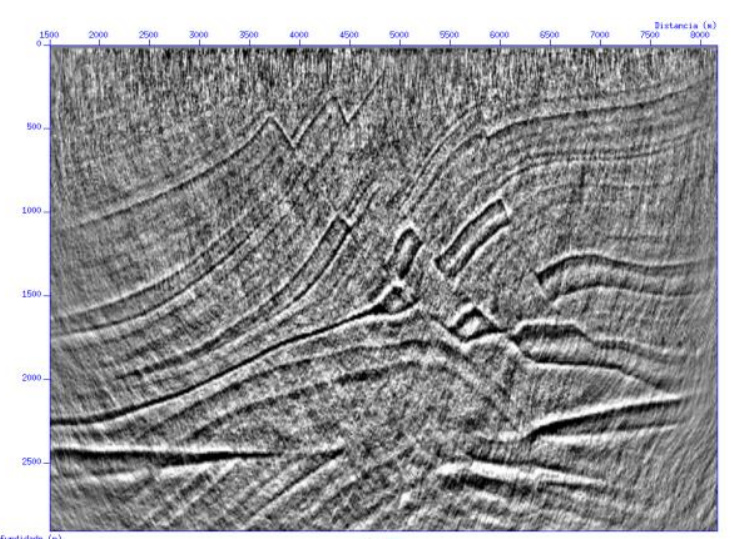

Figura 8- Seção migrada final resultante do empilhamento de todos os afastamentos comuns migrados do dado sísmico Marmousi com a presença de ruído aleatório aditivo.

\section{Conclusões}

Neste resumo expandido, foi apresentada uma alternativa de imageamento em uma geologia complexa, utilizando um processo de superposição de contribuições paraxiais no domínio afastamento comum, tal procedimento representa uma extensão na migração Kirchhoff convencional objetivando potencializar a mesma.

\section{Agradecimentos}

Agradecemos o Laboratório de Ondas Sísmicas do Instituto de Geociências da Universidade Federal do Pará por disponibilizar estações de trabalho, as quais viabilizaram a implementação dos testes numéricos aqui apresentados.

\section{Referências}

COSTA, M. J. S. Migração pré-empilhamento Kirchhoff feixes gaussianos 2.5D nos domínios afastamento comum e ângulo-comum., 2012. 114f. Tese (doutorado)Universidade Federal do Pará, Programa de PósGraduação em Geofísica, Belém, Pará, Brasil.

Ferreira, C.A.S., Cruz, J.C., 2005. Modified Kirchhoff prestack depth migration using the Gaussian beam operator as green function. 9th International Congress of the Brazilian Geophysical Society, Expanded Abstracts.

Ferreira, C. A. S. e Cruz, J. C. R., 2009. Gaussian beam modified true-amplitude diffraction stack migration: Application to Marmousi dataset. Expanded Abstracts of the 11th. SBGF international meeting,Salvador, Brasil.

Garabito, G., Stoffa, P. L., Ferreira, C. A. S., Cruz, J. C. R., 2012. Part II — CRS-beam PSDM: Kirchhoff-beam prestack depth migration using the 2D CRS stacking operator. Journal of Applied Gephysics, vol 85: 102-110. 
Schleicher, J.; Tygel, M.; Hubral, P., 1993. 3D true amplitude finite - offset migration, Geophysics, vol 8:1112 $-1126$.

Sun, Y., Qin, F., Checkles, S., Leveille, J.P., 2000. 3-D prestack Kirchhoff beam migration for depth imaging. Geophysics 65, 1592-1603.

Tygel, M., Schleicher., J., Hubral, P., 1996. An unified approach to 3D seismic reflection imaging - Part II: Theory. Geophysics, vol 61:759-775.

VERSTEEG., R., 1994. The Marmousi experience: Velocity model determination on a synthetic complex data set. The Leading Edge, c. 13:927-936. 